\title{
Extinction as a function of number of reinforced trials and squad composition
}

JAMES H. McHOSE, LARRY L. JACOBY, AND PHILIP A. MEYER SOUTHERN ILLINOIS UNIVERSITY

Number of reinforced trials (40 vs 64), $N_{\mathrm{g}}$ and squad composition ( $M$ is $H$ ) were factorially manipulated. Two $I I$ squads were composed of $S$ s homogeneous with respect to the $N_{g}$ variable while $S$ from the 40 and 64 condition were alternated in running order within $M$ squads. Faster speeds for $M$ as compared with $H$ conditions in extinction were interpreted as reflecting previous reinforcement in $M$ groups of odor cues present in extinction.

Recent data (Ludvigson \& Sytsma, 1967; McHose, 1967) indicate that the patterned running behavior displayed by rats under patterned schedules of reward $(R)$ and nonreward $(N)$ is, at least in part, cued by external stimuli. Differential stimuli are apparently produced on an $\mathbf{R}$ as opposed to an $\mathrm{N}$ event on any one trial, these cues persisting until the next $\mathrm{R}$ or $\mathrm{N}$ event is administered to any $\mathrm{S}$. Thus, depending on the particular trial to trial pattern of reinforcement and the order in which Ss receive trials, these perseverative external stimuli may serve as cues for $\mathbf{R}$ or $\mathrm{N}$ on an immediately subsequent animal trial. With regard to the specific nature of these external cues, McHose \& Ludvigson (1966) suggest that rats exude differential odors on $\mathbf{R}$ as opposed to $\mathrm{N}$ trials, the perseverative traces of which may become cues for $\mathrm{R}$ or $\mathrm{N}$ on a subsequently administered trial, whether the subsequent trial is administered to the $S$ which exuded the odor or to a different $S$.

Assuming only that the resistance to extinction of the running response is increased by reinforcement (in acquisition) of locomotion to cues which are also present in extinction, the olfactory hypothesis may be applied to extinction as well as acquisition phenomena. Thus, if $N$ odors occur on all trials in extinction, reinforcement of locomotion in the presence of $\mathrm{N}$ odors during the acquisition phase should result in increased resistance to extinction relative to that of a condition which receives the same reinforcement schedule, but under which Ss are never reinforced in the presence of $\mathbf{N}$ odors. In the present experiment all groups received continuous reinforcement during the acquisition phase, with extinction trials given at different stages in training, thus providing two comparisons in extinction, each between two continuously reinforced groups, only one of which received reinforcement (in acquisition) for locomotion in the presence of odor cues subsequently present in extinction.

\section{Method}

Subjects were 32 naive, 90 day old male albino rats.
Fourteen days prior to the first experimental day (Day 15), all Ss were placed on a $23 \mathrm{~h}$ food deprivation regimen maintained throughout the experiment. On Days 13 and 14, approximately $1 \mathrm{~g}$ of $45 \mathrm{mg}$ Noyes pellets, identical to the subsequent reinforcement pellet, was incorporated into $S$ 's daily rations.

The runway apparatus was comprised of a gray start, and black alley and goal (GB) sections (cf., McHose \& Ludvigson, 1966, for details). Dropping a door separating the start and alley sections initiated a clock which stopped when $S$ interrupted a photobeam located 6 in. goalward of this door, yielding a start time. Photocell and clock circuitry also provided traversal times over the next $12 \mathrm{in}$, and the last $15 \mathrm{in}$. of the runway (run and goal times, respectively).

A 2 by 2 factorial design was employed with groups of eight Ss designated by squad makeup, homogeneous $(\mathrm{H})$ or mixed $(\mathrm{M})$, and by the number of continuously reinforced acquisition trials received prior to 24 trials of extinction, 40 or 64. All Ss received four trials per day with each running group (squad) of eight Ss receiving all of its daily trials before administration of trials to the next squad. Within a squad, each $S$ received its first daily trial before the first $S$ in the running order recelved its second daily trial, etc. Within two (M) squads, Condition 40 and $64 \mathrm{Ss}$ were alternated in the dally running order. Thus, each M-64 S received each of the last 24 acquisition trials after the preceding $S$ in the running order received an extinction trial and, conversely, each M-24 S received extinction trials following reinforcement for the preceding $\mathrm{S}$. One of the remaining two $(\mathrm{H})$ squads constituted the $\mathrm{H}-40$ condition, and the other the $\mathrm{H}-6+6$ condition. The order in which $\mathrm{S}$ received daily trials within these squads was the same as that of the $M$ squads, i.e., each $S$ received its first daily trial prior to the administration of a second trial to the first $S$ in the running order, etc. On the first day of extinction for Groups M-64 and $H-64$, the remaining $(M-61) S s$ in the two $M$ squads were combined into one squad and the running order of Ss within Group H-64 was rerandomized.

Reinforcement consisted of $20 \mathrm{sec}$ access to $45 \mathrm{mg}$ Noyes pellets. On extinction trials, Ss were confined to the unbaited GB for $20 \mathrm{sec}$, this $20 \mathrm{sec}$ confinement time serving as the sole criterion for removal of $S$ from the apparatus on an extinction trial.

Start, run, and goal times were converted to reciprocals, yielding three speed scores on each trial for each $S$. 


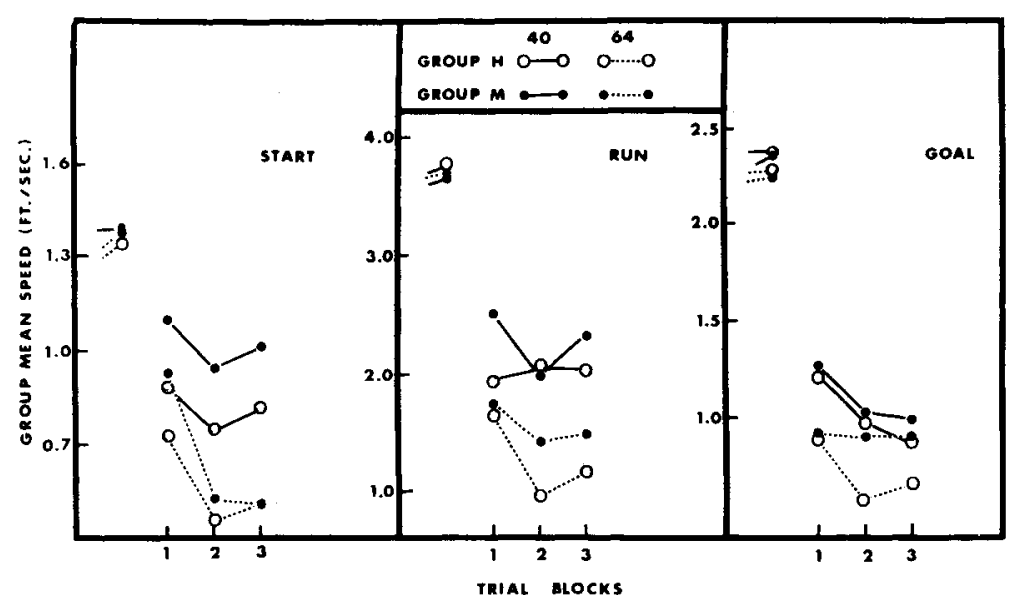

Fig. 1. Speeds in the different alley segments as a function of blocks of trials.

\section{Results}

Group mean speeds in the different segments of the alley by blocks of extinction trials are presented in Fig. 1. The unconnected points represent mean speeds over the last eight acquisition trials. Means for Block 1 exclude speeds on the first nonreinforced trial, successive blocks thus consisting of seven, eight, and eight trials.

As may be seen, at each level of the acquisition training variable $\left(\mathrm{N}_{\mathrm{g}}\right)$, the $M$ group ran faster in extinction than did the $H$ group. Since the faster speeds for the $M$ as compared with the $H$ condition were evidenced at different stages of extinction, depending upon the segment of the alley as well as the level of the $\mathrm{N}_{\mathrm{g}}$ variable, two separate variance analyses were performed on each of the three measures. Three factor (Squad type, $\mathrm{N}_{\mathrm{g}}$, Extinction block) variance analyses yielded significant $(\alpha=.05)$ Squad effects in the start and run measures ( $F=4.33$ and 4.71, respectively, $\mathrm{df}=1 / 28$ ). The Squads effect approached significance in the goal measure ( $F=3.59$, $\mathrm{df}=1 / 28)$. A significant $\mathrm{N}_{\mathrm{g}}$ effect was obtained in each measure ( $F=19.80,28.35$, and 10.96 for start, run, and goal, respectively, df $=1 / 28$ ). Similar variance analyses, in which the Block variable was comprised of the last acquisition and first extinction blocks, yielded a significant squad by Trial Block interaction in the start and run measures ( $F=5.64$ and $8.04, \mathrm{df}=1 / 28$ ) as well as a significant $\mathrm{N}_{\mathrm{g}}$ by Trial Block interaction in the run measure $(F=13.53$, df $=1 / 28$ ).

\section{Discussion}

The present data demonstrate that the resistance to extinction of a continuously reinforced $\mathrm{S}$ depends upon the reinforcement events obtained by other Ss preceding that $S$ in the order of trial administration. Those Ss which recelved reinforcement while preceding adjacent $S s$ in the running sequence wi in squads were extinguished, i.e., Ss constituting Group M-64, were subsequently more resistant to extinction than Ss which received the same amount of acquisition training $(\mathrm{H}-64)$. Similarly, extinction of
Group M-40 while adjacent Ss received reward yielded increased resistance to extinction relative to that of Group $\mathrm{H}-40$ in which adjacent Ss were extinguished at the same time.

The higher speeds in extinction for $M$ as compared with $\mathrm{H}$ groups may be interpreted as the result of differential conditioning, between groups, of the running response to the odor cues present in extinction. Since Ss adjacent in the sequence of trial administration were extinguished at the same time in Groups M-64 and H-64, Ss in both groups were exposed to perseverative $\mathbf{N}$ odors on each extinction trial. Thus, the third $S, S_{3}$ in each group, received each extinction trial in the presence of an $N$ odor exuded by $S_{2}$ of that group, etc. Only Ss in Group M-64, however, were reinforced for locomotion in the presence of $\mathrm{N}$ odors in the acquisition period, resulting in heightened resistance to extinction for this group relative to that of Group $\mathrm{H}-64$. With respect to extinction after 40 trials, Group $M-40$ was extinguished in the presence of $\mathrm{R}$ odors. Thus, Group $\mathrm{M}-40$ alone received reinforcement in acquisition for locomotion in the presence of the odor trail present in extinction.

Finally, it should be noted that the higher speeds in extinction for groups which received 40 acquisition trials relative to those which received 64 trials is consistent with previously reported data which suggest that resistance to extinction in the runway apparatus decreases as the number of reinforced trials increases (cf., Ison, 1962).

\section{References}

ISON, J. R. Resistance to extinction as a function of number of reinforcements. J. exp. Psychol, 1962, 64, 314-317.

LUDVIGSON, H. W., \& SYTSMA, D. The sweet smell of success: Apparent double alternation in the rat. Psychon. Sci., 1967, 9, 283-284.

McHOSE, J. H. Patterned running as a function of the sequence of trial administration. Psychon. Sci., 1967, 9, 281-282.

McHOSE, J. H., \& LUDVIGSON, H. W. Differential conditioning with non-differential reinforcement. Psychon. Sci, 1966, 6, 485-486. Note

1. Supported by Public Health Service Research Grant MH 10340 and $5 \mathrm{~T} 1 \mathrm{MH} 7817$. 